\title{
Placebo and nocebo effects: An introduction to the terms and their presence in oncology
}

\author{
Erald Bakiu' ${ }^{(\mathbb{D}}$, Tolunay Akbaş ${ }^{1}$, Funda İfakat Tengiz ${ }^{2}(\mathbb{D}$ \\ ${ }^{1}$ Izmir Katip Çelebi University Faculty of Medicine, Izmir, Turkey \\ ${ }^{2}$ Department of Medical Education, Izmir Katip Çelebi University Faculty of Medicine, Izmir, Turkey
}

\begin{abstract}
Placebo and nocebo effects are psychobiological effects affecting the homeostasis of the human body, either by curing diseases or by creating and deteriorating pathological states. Despite being a powerful mind-body connection, they have been hidden under the concept of an inert treatment, used in randomized controlled trials, and their true potential has been neglected. When it comes to their presence in oncology, they are responsible for either increasing or reducing pain, anxiety, depression, toxicity and, in quite low rates, tumor size. Further research and implementation of the knowledge of placebo and nocebo effects in medical education will allow harnessing the true potential of these effects and will raise awareness in health professionals that every action they take has a profound effect on their patients.

Keywords: Anxiety, cancer, depression, neoplasm, nocebo, oncology, pain, placebo, positive framing, toxicity.
\end{abstract}

The words "placebo" and "nocebo", the latter being derived from the first, have quite a long and interesting history. Far from the latest definitions in the medical terminology, both words originate from religious jargon, respectively meaning "I shall please" and "I shall harm". ${ }^{[1]}$ In the early years of Christianity, monks used to pray for the deceased members of their communities by reading the ninth verse of Psalm 116 from the Hebrew Bible, which stated "Placebo Domino in regione vivorum," translated as "I shall please the God in the region/land of the ones alive". ${ }^{[2]}$

Ironically, the translation of this verse was erroneous, for its original version, before being translated from Hebrew was, "I shall walk with God in the land of the ones alive", ${ }^{[2]}$ marking the first mistake in using the term of placebo.

In medieval English, the word placebo had rather non-specific meanings including its use as a noun suggesting servility or even in the term "placebo songs" sung by professional mourners who were paid to attend funerals of deceased with insufficient family members ${ }^{[3,4]}$ The word first appeared in medical context in the lectures of William Cullen, a medical educator, in 1772 and later in the 1785 edition of the New Medical Dictionary by George Motherby, defining "placebo" as "a commonplace method or medicine". ${ }^{4]}$ An adequate definition wasn't provided until 1811, when the word appeared in Hooper's Medical Dictionary under the definition, "an epithet given to a medicine adapted more to please than to benefit

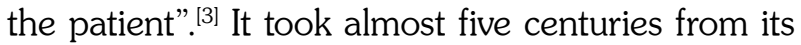
initial use until the word was officially known under its current meaning.

Needless to say, the concepts, placebo and the placebo effect (parallelly, nocebo and the nocebo effect) are related, but different terms. One is

Received: October 25, 2019 Accepted: December 05, 2019 Published online: April 15, 2020

Correspondence: Erald Bakiu. İzmir Katip Çelebi Üniversitesi Tıp Fakültesi, 35620 Çiğli, İzmir, Türkiye.

Tel: +90 545 - 2053992 e-mail: bakiuerald@gmail.com 
used to express an inert substance without any pharmaceutical value and the other is used to express an effect that changes the natural course of a physical condition, may this be by using a placebo/nocebo or not. To elaborate on the last remark, the terms placebo effect and nocebo effect are the roof of many effects that induce deterioration or amelioration of a pathology such as: placebo and nocebo response, the original response that the inert substances are responsible for, spontaneous remission or the natural course of a disease, the effect of concurrent interventions of two agents may them be respectively inert or not, and even the placebo effect of an active treatment). ${ }^{[5-7]}$ Many placebo effects are classified by their effectors as well as many placebo-related effects without any direct connection to inert substances being classified by their psychosocial effectors. In addition, there are also several phenomena that are mistaken for placebo effects such as simple statistical examples of regression to the mean or clinician and patient biases. ${ }^{[7]}$

The first description of the placebo effect was made by Beecher ${ }^{[5]}$ in 1995 who conducted research using meta-analytic methods to extract and process data from 15 randomized clinical trials (RCTs) of prospective pharmaceutical components. ${ }^{[8]} \mathrm{He}$ concluded that $35.2 \pm 2.2 \%$ of the patients subjected to placebos were responsive to them. ${ }^{[5]}$

The work of Beecher was accepted by many authors and with further research, the relieving effects of placebos in pain reduction, depression, anxiety, and cardiovascular diseases have been mentioned. ${ }^{[9]}$ However, his article was criticized by many due to his poor definition of the placebo effect, failure to distinguish the effect from the misleading phenomena, and lack of appropriate documentation in his analysis. ${ }^{[7,6,9]}$

For cancer patients, the most interesting story of the placebo effect is a case report by Bruno Klopfer from his personal communication with Dr. Philip West, a physician in Long Beach California, in 1957. The history of Mr. Wright, whose lymphatic tumors had shrunk with only the power of his mind and the curative effect of placebo while he had only approximately two weeks to live, is quite astonishing. The same Mr. Wright, who after learning that injection with Krebiozen, a potential candidate for cancer treatment at the time, was completely unuseful, was rehospitalized under the nocebo effect and succumbed only a few days later. ${ }^{[10]}$

Several RCTs have been processed with cancer patients, with most of them focusing on the adverse effects of cancer such as nausea and vomiting, malignant pain, anxiety, and depression rather than the parameters of the neoplasm itself. ${ }^{7]}$ During the trials of ondansetron (Zofran) as a medication for nausea and vomiting, a total of 349 patients of 27 oncology centers, who had no prior chemotherapy were divided into four groups and received different doses of the medication and as well placebos. The group receiving placebos, considering treatment failures, had a response rate of $16 \% .{ }^{[1]]}$ In another review of the association between pain and the placebo effect, data from four RCTs showed that among 288 patients, 122 achieved $50 \%$ or greater pain relief from placebos and that these patients were more responsive to the active drug treatment. ${ }^{[12]}$ This study was criticized due to the fact that the evaluation of pain reduction was simply the patient's estimation of the percentage of pain relief. ${ }^{[9]}$

In another RTC, Boureau et al. ${ }^{[13]}$ reported that $51 \%$ of cancer patients with bone metastases were responsive to placebo. The placebo effect lasted seven more days following the 7-day treatment plan. ${ }^{[13,14]}$ Conducted an RCT on alprazolam and reported the placebo and active treatment showed surprisingly similar rates in the depression assessment and only an $11 \%$ difference in the anxiety assessment.

On the other hand, research on the nocebo effect in adverse events is been limited due to ethical reasons and consequently, not much numerical data is available. It has drawn attention that if treatment, may it be active or placebo, is given under a bad context or in a careless manner, can cause adverse events, opening a new topic towards the education of health professionals to be discussed later on.

The purpose of this study is to raise awareness of the strong mind-body connection of how the mind can heal or sicken the body carrying it. We aim to inspire our peers to ponder new possibilities to enhance the mind-body connection and to hopefully discover how one can utilize his/her inner "pharmaceutical factory" to reduce 
unnecessary drug intake. Finally, we hope to point out the therapeutic effects of the health professional himself/herself and clarify that not only the prescribed therapy but also every action taken by the professional has its effect, whether negative or positive.

\section{METHODS}

A computer-based search of articles related to our topics was conducted on the PubMed and DergiPark databases. We encountered a significant number of articles and we selected among them according to their relevance to the topic and their importance judged by their citations. The search continued from these articles to the references of these articles to trace the chronology of the information regarding our topic. After compiling a library, articles requiring expertise on neurosciences, biostatistics, and pharmacology were excluded. The aspects of the topic were divided between researchers for each of them to expand their knowledge on different domains of the topic such as the history of placebo and nocebo, the placebo analgesia, the nocebo hyperalgesia, minor adverse effects in cancer, the placebo effect on neoplasm, and the implementation of the information in medical practice. Cross studies were performed by exchanging topics and having bilateral and then general discussions. We reviewed books, placebo-controlled randomized control trials (double-blinded or not), case studies, and reviews on the topic and carefully inspected the discussed effects on side effects of cancer and the authors' opinions on the phenomena. We meticulously classified authors supporting and referencing each other as well as providing contrasting opinions or statistics. We would like to state that all data were inspected without bias. After presenting all the accumulated information we also shared our novice opinions and prospects on the topic.

\section{RESULTS}

New developments in the field of oncology have made cancer treatment more efficient and reduced the invasion of these treatments in the human body. However, non-specific toxic side-effects still seem to be a major issue. Pain, depression, anxiety, fatigue, nausea and vomiting, dizziness, and irritation constitute the greater portion of these effects. The cause of these effects is often the treatment itself, along with its objective components, although we cannot ignore the fact the patient expectations play an important role in preventing or inducing adverse events. ${ }^{[15]}$

Several biopsychosocial factors are present during treatment, and the balance of these factors include motivation, caring, and trust. When there is a proper balance between these factors, we get the placebo effect, but in the case of instability the nocebo effect would not fail to appear. ${ }^{[16]}$

\section{Pain}

Malignant pain in cancer is closely related to a dynamic duo, consisting of placebo analgesia and nocebo hyperalgesia. Positive or negative expectations for the outcome of the treatment and the extent of the pain, affect different neurochemical systems and divert the flow of hormones, causing the pain to amplify or diminish. ${ }^{[17]}$ The placebo analgesia is associated with the endogenous opioids and this statement is consistent with the findings. ${ }^{[18,19]}$ The studies showed that naloxone enhanced the pain in placebo responders, who were treated with the inert substance before being injected with the opiate antagonist, leaving us with the impression that the opiate-like hormone's activity was inhibited by it. ${ }^{[18,20]}$

In another trial, a hidden administration of morphine (in order to eliminate the placebo effect of the active treatment itself) of $4 \mathrm{mg}$ and $6 \mathrm{mg}$ was proven to be effectively equal with an open administration of intravenous saline (placebo), meaning that it is the same nature of neurotransmitter/hormone that creates this effect and that the placebo analgesia is mediated by endorphins. ${ }^{[19]}$

On the other hand, nocebo hyperalgesia is associated with anxiety as well as anxietyrelated neurochemical processes including cholecystokinin (CCK) signaling in the CKK-ergic systems. The latter facilitates pain transmission leaving us with the reality that even a few words causing tension can be quite painful. As a matter of fact, it was found that the power of the words extend to manipulating the direction of nitrous oxide's action from analgesia to hyperalgesia. ${ }^{[21]}$ Fortunately the CCK-antagonists have been proven to serve as blockers for this 
hyperalgesia, opening new possibilities in pain management and treatment. ${ }^{[17,22,23]}$ However, it is important for health professionals to help patients balance both of these neurochemical systems so that they can experience the most advantageous course possible throughout a painful condition or better response to therapy. ${ }^{[23]}$

When it comes to cancer treatment and the concrete number in that field for pain reduction due to the placebo effect, Chvetzoff and Tannock $^{[9]}$ presented an informative comparison between subjective symptoms and cancer progression in placebo groups. They found that the percentage of patients in placebo groups who reported reduced pain ranged from approximately 0-21\% ${ }^{[9]}$ Another analysis, which was skeptical regarding other symptoms, stated that placebo interventions can influence patientreported pain reduction in certain settings. ${ }^{[24,25]}$

\section{Toxicity}

Until now we have mentioned the placebonocebo effects related to expectancy, addressing the first mechanism of the placebo-nocebo effect. While looking further into another side effect of cancer treatments, toxicity or simply put, nausea or vomiting, we delve into the second mechanism of placebo-nocebo effect: the classical conditioning placebo-nocebo effect.

Administration of cytotoxic drugs, can cause vomiting within two hours and can persist from 2 to 24 hours. Furthermore, there is evidence that patients experience these side effects in anticipation of chemotherapy, even before receiving injections. ${ }^{[26,27]}$ We will explore the placebo effect observed in RCTs of antiemetic drugs followed by the nocebo effect in anticipatory nausea.

The available data on placebo effect can be quite ambiguous due to the fact that it is gathered in trials conducted for a purpose other than measuring the effect. Despite not having data on the natural history of patients who are not exposed to placebo or active treatment, we can distinguish this effect in the trials of several drugs. While active treatment was superior to the placebo in most cases in several RCTs, the effect of the inert treatment is quite considerable. ${ }^{[11,28]}$

The nocebo effect in nausea is the main cause of anticipatory nausea (AN). Stockhorst et al. ${ }^{[26,29-31]}$ studied $\mathrm{AN}$ and its underlying classical (Pavlovian) conditioning and observed that the patients relate the odors, tastes, the sight of the injections, the doctor or nurse as conditioned stimuli with the active drug as an unconditioned stimulus, initiating a chain reaction into a conditioned response which is vomiting. Studies by Stockhurst throughout the years report prevalence of AN ranges from 14 to $63 \%$ and prevalence of anticipatory vomiting (AV) ranges from 9 to 27\%. By creating a distracting effect from the conditioned stimuli, using overshadowing with simple salient solutions, has led to improvement in these side effects. ${ }^{[26,29-32]}$

\section{Psychological effects}

Depression and anxiety are common psychological health issues in cancer patients. The placebo effect on patients with these conditions has been measured in RCTs of potential medications. We mentioned earlier the RCT comparing alprazolam and placebo and the active treatment effects on anxiety and depression. In another meta-analytic review, Walsh et al. ${ }^{[33]}$ collected data from 75 RCTs of antidepressants and found that $29.7 \%$ of placebo group patients and $50.1 \%$ of patients receiving active treatment showed decrease in symptoms.

The nocebo effect was also mentioned earlier regarding nocebo hyperalgesia, with anxiety as a key factor, but the most striking examples were described by Cannon. ${ }^{[34,35]}$ In his article, he illustrated examples of "voodoo" deaths worldwide, such as people dying from the anxiety and depression of being cursed by a shaman or the chieftain of their tribe. Examples of "malignant anxiety" were also given to explain how patients could literally die due to peak levels of anxiety.

\section{Tumor desponse}

The most considerable effect expected by treatment for cancer is the reduction of neoplasm. While there are shocking reports from isolated examples, according to a review by Chvetzoff and Tannock, ${ }^{[9]}$ out of all the trials that evaluated the unbiased response of patients, the overall response rate of placebo was $2.7 \%$ (10 of 375 patients). This rate may be as well as simple spontaneous remission. 
A recent review of placebo response in medullary thyroid cancer patients showed that partial placebo response with a shrinkage of more than $30 \%$ of the tumor size was observed in $6.4 \%$ of the patients $(n=211)$ and that a controlled disease status, or non-developing tumor, was achieved by $60.2 \%$ of the patients. ${ }^{[36]}$

\section{DISCUSSION}

As the knowledge of the human body expands, more people start to agree with the idea that a person is not just a single individual but a community. This community consists of up to 40 trillion cells, and while they are mostly controlled by the mind, they are believed to have their own will. In cases of "rebellions", we have negative results such as autoimmune diseases or cancer.

The connections between the mind and the body can be either conscious or unconscious, but despite their type, they have a profound meaning on our existence. Several hypotheses have emerged and one of them is that the mind can heal the body, no matter how poor the body's condition may be. To elaborate, for every medication, there is a receptor for it to interact with, and if there is a receptor for a certain chemical compound in the human body, it is speculated whether or not there is also an endogenous compound similar or with similar effect to the one we obtain through medication. If such a thing exists, are we just blinding the pathway of that compound by using an external source for our needs? Are we getting in the way of human evolution by oversimplifying our lives?

The placebo and nocebo effects are perceived as defense mechanisms developed by the mind and body to deal with external and internal threats. As per our topic, cancer is an internal threat, despite having some external factors. Studies on the placebo effect in oncology are limited and the available data are from trials trying to validate the efficacy of a medication rather than study the placebo effect itself. In order to form a healthy opinion on placebo effects in oncology, trials with a natural history control group must be studied parallel to the placebo group, and when administering the placebo, the context and the design of the trial should be favorable to placebo response. The hierarchy of the placebos should be determined and the administrator should also aim for the effect by creating a productive environment. Doctors and nurses are able to transmit a considerable amount of information to the patient through their words, attitudes, and behavior. Physicians have long known the powerful effect of their relationship with their patients and what is expected from them is to apply this in their practice. ${ }^{[37]}$ It is difficult to determine whether this disregard of studying the placebo effect comes from the fact that it has been deemed unimportant, or that the positive thinking cannot be packed up and sold for a price.

The study of nocebo effects in oncology has been limited only with the non-specific side effects caused by the cytotoxic treatment, while the nocebo effect on the neoplasm itself is unknown. Due to ethical reasons, research regarding the possibility that a physician can induce an increase in the size of the patient's neoplasm would be unacceptable.

The main issue surrounding these phenomena is lack of awareness. There is a critical need for educating clinicians and patients about the effects of placebo-nocebo processes, including how, when, and why they occur. ${ }^{[38]}$

Educating clinicians on placebo effects allows them to utilize these effects while planning treatment or even encourage them to keep a personal record of the patient including the history of non-specific factors with positive outcomes on the patient's condition allowing more accessible records of placebo effects and placebo responders. ${ }^{[39]}$ As a bonus, health professionals who emphasize treatment efficacy and improving treatment knowledge can raise expectancies and induce the placebo effect in patients. ${ }^{[40]}$

On the other hand, education on the nocebo effect is vital to sharing delicate information regarding a condition. If information about a condition can promote sickness by creating bad expectations, then one must be careful in both public health communications and in clinical medicine. ${ }^{[41]}$ Clinicians must learn to educate their patients while extracting the patient's expectations so that the delivery of information can be properly adapted to the needs of the patient. ${ }^{[38]}$ Studies suggest that positive framing of side effect warnings is a promising technique for reducing nocebo side effects, while maintaining informed consent. ${ }^{[42]}$ 
In order to achieve this kind of education, curricula for the preparation of future clinicians should include elements from neurobiology, psychobiology, and last but not least, communication lectures on transmitting information in positive framing.

\section{Declaration of conflicting interests}

The authors declared no conflicts of interest with respect to the authorship and/or publication of this article.

\section{Funding}

The authors received no financial support for the research and/or authorship of this article.

\section{REFERENCES}

1. Lemoine P. The placebo mystery or neurobiology of the soul. Bull Acad Natl Med 2011;195:146576.

2. Moerman, D. Meaning, medicine, and the "placebo effect'. New York: Cambridge University Press; 2002.

3. Shapiro AK. A contribution to a history of the placebo effect. Behavioral Science 2007;5:109-35.

4. Kerr CE, Milne I, Kaptchuk TJ. William Cullen and a missing mind-body link in the early history of placebos. J R Soc Med 2008;101:89-92.

5. Beecher HK. The powerful placebo. J Am Med Assoc 1955;159:1602-6

6. Kaptchuk TJ. Intentional ignorance: a history of blind assessment and placebo controls in medicine. Bull Hist Med 1998;72:389-433.

7. Benedetti, F. Placebo effects understanding the mechanisms in health and disease. Oxford: Oxford University Press; 2009.

8. Kaptchuk TJ. Powerful placebo: the dark side of the randomised controlled trial. Lancet 1998;351:1722-5.

9. Chvetzoff G, Tannock IF. Placebo Effects in Oncology. JNCI 2003;95:19-29.

10. Klopfer B. Psychological variables in human cancer. J Proj Tech 1957;21:331-40.

11. Beck TM, Ciociola AA, Jones SE, Harvey WH, Tchekmedyian NS, Chang A, et al. Efficacy of oral ondansetron in the prevention of emesis in outpatients receiving cyclophosphamide-based chemotherapy. The Ondansetron Study Group. Ann Intern Med 1993;118:407-13.

12. Moertel CG, Taylor WF, Roth A, Tyce FA. Who responds to sugar pills? Mayo Clin Proc 1976;51:96- 100.

13. Boureau F, Leizorovicz A, Caulin F. The placebo effect in bone metastatic pain. Presse Med 1988;17:1063-6.

14. Wald TG, Kathol RG, Noyes R Jr, Carroll BT, Clamon GH. Rapid relief of anxiety in cancer patients with both alprazolam and placebo. Psychosomatics 1993;34:324-32.
15. Garg AK. Nocebo side-effects in cancer treatment. Lancet Oncol 2011;12:1181-2.

16. Spiegel H. Nocebo: the power of suggestibility. Prev Med 1997;26:616-21.

17. Atlas LY, Wager TD. How expectations shape pain. Neurosci Lett. 2012;520:140-8.

18. Levine JD, Gordon NC, Fields HL. The mechanism of placebo analgesia. Lancet 1978;2:654-7.

19. Levine JD, Gordon NC, Smith R, Fields HL. Analgesic responses to morphine and placebo in individuals with postoperative pain. Pain 1981;10:379-89.

20. Schafer SM, Geuter S, Wager TD. Mechanisms of placebo analgesia: A dual-process model informed by insights from cross-species comparisons. Prog Neurobiol 2018;160:101-22.

21. Dworkin SF, Chen AC, LeResche L, Clark DW. Cognitive reversal of expected nitrous oxide analgesia for acute pain. Anesth Analg 1983;62:1073-7.

22. Benedetti F, Lanotte M, Lopiano L, Colloca L. When words are painful: unraveling the mechanisms of the nocebo effect. Neuroscience 2007;147:260-71.

23. Colloca L, Benedetti F. Nocebo hyperalgesia: how anxiety is turned into pain. Curr Opin Anaesthesiol 2007;20:435-9.

24. Hróbjartsson A, Gøtzsche PC. Is the placebo powerless? Update of a systematic review with 52 new randomized trials comparing placebo with no treatment. J Intern Med 2004;256:91-100.

25. Hróbjartsson A, Gøtzsche PC. Placebo interventions for all clinical conditions. Cochrane Database Syst Rev 2010;1:CD003974.

26. Stockhorst U, Klosterhalfen S, Klosterhalfen W, Winkelmann M, Steingrueber HJ. Anticipatory nausea in cancer patients receiving chemotherapy: classical conditioning etiology and therapeutical implications. Integr Physiol Behav Sci 1993;28:177-81.

27. Nesse RM, Carli T, Curtis GC, Kleinman PD. Pretreatment nausea in cancer chemotherapy: a conditioned response? Psychosom Med 1980;42:33-6.

28. Moertel CG, Reitemeier RJ, Gage RP. A controlled clinical evaluation of antiemetic drugs. JAMA 1963;186:116-8.

29. Stockhorst U, Wiener JA, Klosterhalfen S, Klosterhalfen W, Aul C, Steingrüber HJ. Effects of overshadowing on conditioned nausea in cancer patients: an experimental study. Physiol Behav 1998;64:743-53.

30. Stockhorst U, Enck P, Klosterhalfen S. Role of classical conditioning in learning gastrointestinal symptoms. World J Gastroenterol 2007;13:3430-7.

31. Stockhorst U, Hall G, Enck P, Klosterhalfen S. Effects of overshadowing on conditioned and unconditioned nausea in a rotation paradigm with humans. Exp Brain Res 2014;232:2651-64.

32. Kamen C, Tejani MA, Chandwani K, Janelsins M, Peoples AR, Roscoe JA, et al. Anticipatory nausea 
and vomiting due to chemotherapy. Eur J Pharmacol 2014;722:172-9.

33. Walsh BT, Seidman SN, Sysko R, Gould M. Placebo response in studies of major depression: variable,substantial, and growing. JAMA 2002;287:1840-7.

34. Cannon WB. "Voodoo" death. American Anthropologist 1942;44:169-81.

35. Cannon WB. "Voodoo" death. American Anthropologist, 1942;44(new series):169-181. Am J Public Health. 2002;92:1593-6.

36. Llavero-Valero M, Guillén-Grima F, Zafon C, Galofré JC. The placebo effect in thyroid cancer: a metaanalysis. Eur J Endocrinol 2016;174:465-72.

37. Balint $\mathrm{M}$. The doctor, his patient, and the illness. Lancet 1955;268:683-8

38. Colloca L. Tell me the truth and 1 will not be Harmed: Informed consents and nocebo effects. Am J Bioeth
2017;17:46-8.

39. Chaput de Saintonge DM, Herxheimer A. Harnessing placebo effects in health care. Lancet 1994;344:995-8.

40. Heisig SR, Shedden-Mora MC, von Blanckenburg P, Rief W, Witzel I, Albert US, et al. What do women with breast cancer expect from their treatment? Correlates of negative treatment expectations about endocrine therapy. Psychooncology 2016;25:1485-92.

41. Hahn RA. The nocebo phenomenon: concept,evidence, and implications for public health. Prev Med 1997;26:607-11.

42. Barnes K, Faasse K, Geers AL, Helfer SG, Sharpe L, Colloca L, et al. Can Positive Framing Reduce Nocebo Side Effects? Current Evidence and Recommendation for Future Research. Front Pharmacol 2019;10:167. 